\title{
THE HAUSDORFF DIMENSION OF GRAPHS OF DENSITY CONTINUOUS FUNCTIONS
}

\author{
ZOLTÁN BUCZOLICH AND KRZYSZTOF OSTASZEWSKI
}

(Communicated by Andrew M. Bruckner)

\begin{abstract}
The density topology on the real line consists of all measurable sets whose all points are their points of Lebesgue density one. A real-valued function of a real variable that is continuous with respect to the density topology on both the domain and the range is termed density continuous. By constructing graphs of density continuous functions as invariant sets of systems of affine maps on the unit square we show that the Hausdorff dimensions of graphs of density continuous functions vary continuously between one and two.
\end{abstract}

The density topology on the real line consists of all measurable sets $E$ such that every point of $E$ is its Lebesgue density point (see Oxtoby [13, p: 90]). It is a completely regular refinement of the natural topology.

The functions $f: \mathbf{R} \rightarrow \mathbf{R}$ which are continuous with respect to the density topology on the domain and the range are termed density continuous.

Bruckner [2] and Niewiarowski [10] investigate homeomorphisms of the real line whose inverses are density continuous. Ostaszewski [11,12] studies local behavior of density continuous functions. Ciesielski and Larson [4], and independently Burke [3], show that all analytic functions are density continuous.

Ciesielski, Larson, and Ostaszewski [7] show that all density continuous functions are in Darboux Baire ${ }^{*} 1$ class, and the class of continuous, density continuous functions is of the first category in the space of continuous functions, equipped with the topology of uniform convergence. In fact, Ciesielski, Larson, and Ostaszewski [6] show that the class of density continuous functions when equipped with the topology of uniform convergence, is of the first category in itself. Thus the space of density continuous functions is, topologically speaking, rather small. On the other hand, it does include most functions studied in calculus.

We will show that despite the rarity of density continuous functions among the continuous functions, there are enough of them to produce graphs of all

Received by the editors February 16, 1990.

1980 Mathematics Subject Classification (1985 Revision). Primary 28A75; Secondary 58F11.

Key words and phrases. Hausdorff dimension, self-affine functions, density topology, density continuity.

This work was completed while the first author visited the University of California at Davis.

The second author had partial support from a University of Louisville research grant. 
possible Hausdorff dimensions for real functions of a real variable.

Let $I=[0,1]$. Malý [8] constructs a density continuous function $f: I \rightarrow I$ such that there exists a set $A$ of measure zero with $|f(A)|=1$. The function $f$ is actually the $x$-coordinate of a Peano area-filling curve. As an illustration to the proof of Theorem 2 we will first recall Maly's construction and show that the function constructed has a graph of the Hausdorff dimension 1.5.

First let $g:[0,9] \rightarrow[0,3]$ be defined as follows: $g(0)=0, g(1)=1$, $g(2)=0, g(3)=1, g(4)=2, g(5)=1, g(6)=2, g(7)=3, g(8)=2$, $g(9)=3$, and let $g$ be linear in each interval of the form $[i, i+1]$ where $0 \leq i \leq 8$. Define

$$
f_{1}(x)=\frac{1}{3} g\left(\frac{1}{9} x\right) .
$$

For every $n \in \mathbf{N}, k \in \mathbf{N}, 0 \leq k<9^{n}, 0 \leq t \leq 9^{-n}$ let

$$
f_{n+1}\left(\frac{k}{9^{n}}+t\right)=f_{n}\left(\frac{k}{9^{n}}\right)+\frac{1}{3} g\left(9^{n+1} t\right)\left(f_{n}\left(\frac{k+1}{9^{n}}\right)-f_{n}\left(\frac{k}{9^{n}}\right)\right) .
$$

Malý [8] shows that the sequence $\left\{f_{n}\right\}_{n \in \mathbf{N}}$ converges uniformly to a continuous, density continuous function $f: I \rightarrow I$. Note that Ciesielski, Larson, and Ostaszewski [7] show that the function constructed by Malý is nowhere approximately differentiable.

The graph of the function $f$ may also be realized as the invariant set of an iterated system (see [1, p. 82]) of nine affine maps of the plane. In fact, let

$$
\begin{aligned}
\phi_{1}\left[\begin{array}{l}
x \\
y
\end{array}\right] & =\left[\begin{array}{ll}
\frac{1}{9} & 0 \\
0 & \frac{1}{3}
\end{array}\right]\left[\begin{array}{l}
x \\
y
\end{array}\right], \\
\phi_{2}\left[\begin{array}{l}
x \\
y
\end{array}\right] & =\left[\begin{array}{ll}
-\frac{1}{9} & 0 \\
0 & \frac{1}{3}
\end{array}\right]\left[\begin{array}{l}
x \\
y
\end{array}\right]+\left[\begin{array}{l}
\frac{2}{9} \\
0
\end{array}\right], \\
\phi_{3}\left[\begin{array}{l}
x \\
y
\end{array}\right] & =\left[\begin{array}{ll}
\frac{1}{9} & 0 \\
0 & \frac{1}{3}
\end{array}\right]\left[\begin{array}{l}
x \\
y
\end{array}\right]+\left[\begin{array}{l}
\frac{2}{9} \\
0
\end{array}\right], \\
\phi_{4}\left[\begin{array}{l}
x \\
y
\end{array}\right] & =\left[\begin{array}{ll}
\frac{1}{9} & 0 \\
0 & \frac{1}{3}
\end{array}\right]\left[\begin{array}{l}
x \\
y
\end{array}\right]+\left[\begin{array}{l}
\frac{1}{3} \\
\frac{1}{3}
\end{array}\right], \\
\phi_{5}\left[\begin{array}{l}
x \\
y
\end{array}\right] & =\left[\begin{array}{ll}
-\frac{1}{9} & 0 \\
0 & \frac{1}{3}
\end{array}\right]\left[\begin{array}{l}
x \\
y
\end{array}\right]+\left[\begin{array}{l}
\frac{5}{9} \\
\frac{1}{3}
\end{array}\right], \\
\phi_{6}\left[\begin{array}{l}
x \\
y
\end{array}\right] & =\left[\begin{array}{ll}
\frac{1}{9} & 0 \\
0 & \frac{1}{3}
\end{array}\right]\left[\begin{array}{l}
x \\
y
\end{array}\right]+\left[\begin{array}{l}
\frac{5}{9} \\
\frac{1}{3}
\end{array}\right], \\
\phi_{7}\left[\begin{array}{l}
x \\
y
\end{array}\right] & =\left[\begin{array}{ll}
\frac{1}{9} & 0 \\
0 & \frac{1}{3}
\end{array}\right]\left[\begin{array}{l}
x \\
y
\end{array}\right]+\left[\begin{array}{l}
\frac{2}{3} \\
\frac{2}{3}
\end{array}\right], \\
\phi_{8}\left[\begin{array}{l}
x \\
y
\end{array}\right] & =\left[\begin{array}{cc}
-\frac{1}{9} & 0 \\
0 & \frac{1}{3}
\end{array}\right]\left[\begin{array}{l}
x \\
y
\end{array}\right]+\left[\begin{array}{l}
\frac{2}{3} \\
\frac{2}{3}
\end{array}\right], \\
\phi_{9}\left[\begin{array}{l}
x \\
y
\end{array}\right] & =\left[\begin{array}{ll}
\frac{1}{9} & 0 \\
0 & \frac{1}{3}
\end{array}\right]\left[\begin{array}{l}
x \\
y
\end{array}\right]+\left[\begin{array}{l}
\frac{8}{9} \\
\frac{2}{3}
\end{array}\right] .
\end{aligned}
$$


Let $G$ stand for the graph of the function $f$. Then

$$
G=\bigcup_{i=1}^{9} \phi_{i}(G) .
$$

We will denote by $\operatorname{dim}(E)$ the Hausdorff dimension of a set $E$ in a Euclidean space.

McMullen [9] determines the Hausdorff dimension of a family of planar sets which are generalizations of the classical Cantor set in $\mathbf{R}$. His main result is:

Theorem 1. Given positive integers $n \geq m$ and a set $R$ consisting of pairs of integers $(i, j)$ with $0 \leq i<n$ and $0 \leq j<m$, define the set $\bar{R}$ by

$$
\bar{R}=\left\{\left(\sum_{k=1}^{+\infty} \frac{x_{k}}{n^{k}}, \sum_{k=1}^{+\infty} \frac{y_{k}}{m^{k}}\right):\left(x_{k}, y_{k}\right) \in R \forall k \in \mathbf{N}\right\} .
$$

Then the Hausdorff dimension of $\bar{R}$ is given by

$$
\operatorname{dim}(\bar{R})=\log _{m}\left(\sum_{j=0}^{m-1} t_{j}^{\left(\log _{n} m\right)}\right)
$$

where $t_{j}$ is the number of $i$ such that $(i, j) \in R$.

Proof. McMullen [9, Theorem, p. 1].

Note that $\bar{R}$ is the invariant set of the iterated function system (see [1, p. 82]) $f_{1}, f_{2}, \ldots, f_{r}$, where $r$ is the number of elements of $R$, and each $f_{i}$ is an affine map contracting the plane by a factor of $n$ horizontally and $m$ vertically. In other words

$$
\bar{R}=\bigcup_{i=1}^{r} f_{i}(\bar{R})
$$

for those affine maps.

Corollary 1. The Hausdorff dimension of the first coordinate function of the Peano area-filling curve (i.e., the function $f$ defined above) equals 1.5 .

Proof. In Theorem 1, let $n=9, m=3, r=9$. It follows from Theorem 1 (and the discussion of its equivalent version above) that the Hausdorff dimension of $G$ equals

$$
\log _{3}\left(\sum_{j=0}^{2} e^{\log _{9} 3}\right)=1.5 .
$$

We will now proceed to prove that a construction similar to the one above can produce a density continuous function with a graph of any desired dimension. We will start by noticing that it suffices to show that Hausdorff dimensions of graphs of density continuous functions form a dense subset of the interval $[1,2]$. 
Lemma 1. If $E_{n}$ is a sequence of subsets of a Euclidean space then

$$
\operatorname{dim}\left(\bigcup_{n \in \mathbf{N}} E_{n}\right)=\sup _{n \in \mathbf{N}} \operatorname{dim}\left(E_{n}\right)
$$

Proof. This can be verified easily using [14, Theorem 9, p. 17].

Corollary 2. If the set of dimensions of graphs of density continuous functions is dense in [1,2] then it equals the entire interval [1,2].

Proof. Let $s \in[1,2]$. Assume that $\left\{s_{n}\right\}_{n \in \mathbf{N}}$ is an increasing sequence of dimensions of graphs of density continuous functions $f_{n}$, such that $\lim _{n \rightarrow \infty} s_{n}=$ $s$. Without loss of generality we may assume that each $f_{n}$ is a surjective mapping of $[0,1]$ onto $[0,1]$ such that $f_{n}(0)=0$ and $f_{n}(1)=1$. Define

$$
f(x)=\frac{1}{2^{n+1}} f_{n}\left(2^{n+1}\left(x-\left(\frac{1}{2}+\frac{1}{4}+\cdots+\frac{1}{2^{n}}\right)\right)\right)+\frac{1}{2}+\frac{1}{2}+\cdots+\frac{1}{2^{n}}
$$

for

$$
\frac{1}{2}+\frac{1}{2}+\cdots+\frac{1}{2^{n}} \leq x \leq \frac{1}{2}+\frac{1}{2}+\cdots+\frac{1}{2^{n}}+\frac{1}{2^{n+1}},
$$

and

$$
f(x)=2 f_{1}\left(\frac{1}{2} x\right)
$$

for

$$
0 \leq x \leq \frac{1}{2} \text {. }
$$

Then $f$ is density continuous and the Hausdorff dimension of its graph equals $s$ by Lemma 1 .

Theorem 2. The set of Hausdorff dimensions of graphs of surjective density continuous functions $f:[0,1] \rightarrow[0,1]$ is dense in $[1,2]$.

Proof. Let $n \in \mathbf{N}$ be arbitrary, $0 \leq l \leq n$, and $m \in \mathbf{N}$ be odd. Define the following maps of the unit square into itself:

$$
\begin{aligned}
& \phi_{1}\left[\begin{array}{l}
x \\
y
\end{array}\right]= {\left[\begin{array}{cc}
\frac{1}{m n} & 0 \\
0 & \frac{1}{n}
\end{array}\right]\left[\begin{array}{l}
x \\
y
\end{array}\right], } \\
& \phi_{2}\left[\begin{array}{l}
x \\
y
\end{array}\right]=\left[\begin{array}{cc}
-\frac{1}{m n} & 0 \\
0 & \frac{1}{n}
\end{array}\right]\left[\begin{array}{l}
x \\
y
\end{array}\right]+\left[\begin{array}{c}
\frac{2}{m n} \\
0
\end{array}\right], \\
& \phi_{3}\left[\begin{array}{l}
x \\
y
\end{array}\right]=\phi_{1}\left[\begin{array}{l}
x \\
y
\end{array}\right]+\left[\begin{array}{c}
\frac{2}{m n} \\
0
\end{array}\right], \\
& \phi_{4}\left[\begin{array}{l}
x \\
y
\end{array}\right]=\phi_{2}\left[\begin{array}{l}
x \\
y
\end{array}\right]+\left[\begin{array}{c}
\frac{2}{m n} \\
0
\end{array}\right], \\
& \vdots \\
& \phi_{m}\left[\begin{array}{l}
x \\
y
\end{array}\right]=\phi_{m-2}\left[\begin{array}{l}
x \\
y
\end{array}\right]+\left[\begin{array}{c}
\frac{2}{m n} \\
0
\end{array}\right] .
\end{aligned}
$$


Furthermore, let

$$
\begin{aligned}
\phi_{m+1}\left[\begin{array}{l}
x \\
y
\end{array}\right] & =\phi_{1}\left[\begin{array}{l}
x \\
y
\end{array}\right]+\left[\begin{array}{c}
\frac{1}{n} \\
\frac{1}{n}
\end{array}\right], \\
\vdots & \\
\phi_{r m+j}\left[\begin{array}{l}
x \\
y
\end{array}\right] & =\phi_{j}\left[\begin{array}{l}
x \\
y
\end{array}\right]+\left[\begin{array}{c}
\frac{r}{n} \\
\frac{r}{n}
\end{array}\right], \quad(1 \leq r \leq l-1,1 \leq j \leq m) .
\end{aligned}
$$

Consider now the invariant set of the above affine functions system. It is not necessarily the graph of a function defined on $[0,1]$; however, it will become one if we add to it the line segment connecting the points $(l / n, l / n)$ and $(1,1)$, and the countable collection of its images under maps of the form $\phi_{i_{1}} \circ \phi_{i_{2}} \circ$ $\cdots \circ \phi_{i_{N}}$, where $i_{j}$ is an integer between 1 and $\operatorname{lm}$ for each $j=1,2, \ldots, N$. Denote by $f$ the function obtained above. One can easily visualize $f$ as a uniform limit of the following continuous, piecewise linear functions $\left\{f_{N} \mid N \in\right.$ $\mathbf{N}\}$. Denote $f_{0}(x)=x$ for $x \in[0,1]$, and let $f_{N}:[0,1] \rightarrow[0,1], N \in \mathbf{N}$ be the function whose graph is the union of the images of the graph of $f_{0}$ under the mappings $\phi_{i_{1}} \circ \phi_{i_{2}} \circ \cdots \circ \phi_{i_{N}}$, where $i_{j}$ is an integer between 1 and $\operatorname{lm}$ for each $j=1,2, \ldots, N$, and which is linear in the intervals where the above definition does not extend (i.e., we gradually add the linear "pieces" appearing in the graph of $f$ ). It is easy to verify the uniform convergence of $f_{N}$ to $f$. This implies that $f$ is continuous.

We claim that for any measurable $E \subset[0,1], f^{-1}(E)$ has the same measure as $E$.

We shall prove that $\left|f^{-1}(I)\right|=|I|$ for $I$ of the form $\left(v / N^{k},(v+1) / N^{k}\right)$, $v, N, k \in \mathbf{N}$. Since intervals of this form generate the $\sigma$ algebra of measurable sets in $[0,1]$ this will imply that for any measurable set $E$ we have $\left|f^{-1}(E)\right|=$ $|E|$. Obviously $\left|f_{0}^{-1}(I)\right|=|I|$ for any interval $I$. Observe that $f_{1}(x)=x$ for any $x \in[l / n, 1]$. Thus if $I \subset[l / n, 1]$ then $\left|f_{1}^{-1}(I)\right|=|I|$. One can easily see that $f_{1}$ is mapping each interval of the form $[(r-1) / n, r / n], r=1,2, \ldots, l$ into itseif. If $I=\left((v-1) / n^{k}, v / n^{k}\right)$ and $(r-1) / n<v / n^{k} \leq r / n$ then $f_{1}^{-1}(I)$ will be a subset of $[(r-1) / n, r / n]$. In fact $f_{1}^{-1}(I)=\bigcup_{j=1}^{m} \phi_{r m+j}^{-1}(I)$. It is easy to check that $\left|f_{1}^{\prime}(x)\right|=m$ if $0 \leq x \leq l / n$ and $x \neq t / n m$ for a $t \in \mathbf{N}$. This implies that $\left|\phi_{r m+j}^{-1}(I)\right|=|I| / m$ for $j=1, \ldots, m$. Thus $\left|f_{1}^{-1}(I)\right|=m \cdot|I| / m=|I|$. A similar, slightly more complicated argument can show that $\left|f_{N}^{-1}(I)\right|=|I|$ for every $N \in \mathbf{N}$.

Recall that the definition of the functions $f_{N}$ implies that $f_{N-1}$ maps an interval of the form $\left[(s-1) /(n m)^{N-1}, s /(n m)^{N-1}\right]$ into an interval of the form $\left[(t-1) / n^{N-1}, t / n^{N-1}\right], t, s \in \mathbf{N}$ if and only if $f_{n}$ is also mapping $\left[(s-1) /(n m)^{N-1}, s /(n m)^{N-1}\right]$ into $\left[(t-1) / n^{N-1}, t / n^{N-1}\right]$. That is, the graph 
of $f_{N}$ and in fact the graphs of $f_{M}, M \geq N$ on the interval $\left[(s-1) /(n m)^{N-1}\right.$, $\left.s /(n m)^{N-1}\right]$ will remain in the rectangle $\left[(s-1) /(n m)^{N-1}, s /(n m)^{N-1}\right] \times$ $\left[(t-1) / n^{N-1}, t / n^{N-1}\right]$. Observe also that for any $y \in \mathbf{R}, N \in \mathbf{N}$ the set $f_{N}^{-1}(y)$ is finite. Therefore $f_{N}^{-1}(I)$ equals $f_{k}^{-1}(I) \backslash\left(f_{N}^{-1}\left((v-1) / n^{k}\right) \cup f_{N}^{-1}\left(v / n^{k}\right)\right)$ if $N \geq$ $k$ and $I=\left((v-1) / n^{k}, v / n^{k}\right)$. Since $f=\lim _{N \rightarrow \infty} f_{N}$ we obtain that $f^{-1}(I)$ equals $f_{k}^{-1}(I)$ minus countably many points and hence $\left|f^{-1}(I)\right|=\left|f_{k}^{-1}(I)\right|$. This, together with $\left|f_{k}^{-1}(I)\right|=|I|$ implies that $\left|f^{-1}(I)\right|=|I|$ and proves our claim.

The claim in turn implies that $f$ is density continuous.

Now we will show that by varying the parameters $m, n, k$ we can have the Hausdorff dimension of the graph of $f$ in any subinterval of $(1,2]$ (note that $f(x)=x$ is density continuous and its graph has dimension 1 ).

In fact, let $G$ be exactly the invariant set of the iterative function system $\phi_{1}, \phi_{2}, \ldots, \phi_{l m}$ (i.e., without the linear pieces added). The Hausdorff dimension of the linear pieces equals one. Thus the Hausdorff dimension of the graph of $f$ equals the maximum of the two numbers: one and $\operatorname{dim}(G)$.

Theorem 1 implies that the Hausdorff dimension of $G$ equals

$$
\log _{n} \sum_{j=0}^{l-1} m^{\log _{m n} n}
$$

as the functions $\phi_{j}$ are contracting the plane by a factor of $\mathrm{nm}$ horizontally and $n$ vertically; $t_{j}=m$ for $j=0,1, \ldots, l-1$ and $t_{j}=0$ otherwise. Let $d$ denote the unknown dimension of the set $G$. A simple calculation gives

$$
d=\frac{\ln l}{\ln n}+\frac{\ln m}{\ln m+\ln n} \text {. }
$$

Obviously

$$
\left\{\frac{\ln l}{\ln n}: n \in \mathbf{N}, l \leq n, l \in \mathbf{N}\right\}
$$

is dense in $[0,1]$. Since

$$
\lim _{m \rightarrow \infty} \frac{\ln m}{\ln n+\ln m}=1
$$

we conclude that

$$
[1,2] \cap\left\{\frac{\ln l}{\ln n}+\frac{\ln m}{\ln m+\ln n}: n, m \in \mathbf{N}, l \leq n, l \in \mathbf{N}\right\}
$$

is dense in $[1,2]$. This completes the proof of Theorem 2.

\section{REFERENCES}

1. M. Barnsley, Fractals everywhere, Academic Press, 1988.

2. A. M. Bruckner, Density-preserving homeomorphisms and the theorem of Maximoff, Quart. J. Math. Oxford (2) 21 (1970), 337-347. 
3. M. Burke, Some remarks on density-continuous functions, Real Anal. Exchange 14 (1988$89), 235-242$.

4. K. Ciesielski and L. Larson, The space of density continuous functions, Acta Math. Hung. (to appear).

5. K. Ciesielski, L. Larson, and K. Ostaszewski, Density continuity versus continuity, Forum Math. 2 (1990), 265-275.

6. __ Four continuities on the real line, submitted.

7. __ Differentiability and density continuity, Real Anal. Exchange 15 (1) (1989-90), 239248.

8. J. Malý, The Peano curve and the density topology, Real Anal. Exchange 5 (1979-80), 326329.

9. C. McMullen, The Hausdorff dimension of general Sierpiniski carpets, Nagoya Math. J. 96 (1984), 1-9.

10. J. Niewiarowski, Density-preserving homeomorphisms, Fund. Math. 106 (1980), 77-87.

11. K. Ostaszewski, Continuity in the density topology, Real Anal. Exchange 7 (2) (1982), 259270.

12. $\frac{}{414}$, Continuity in the density topology II, Rend. Circ. Mat. Palermo (2) 32 (1983), 398414.

13. J. C. Oxtoby, Measure and category, Springer-Verlag, New York, 1971.

14. C. A. Rogers, Hausdorff measures, Cambridge University Press, 1970.

Department of ANalysis, Eötvös Loránd University, Budapest, Múzeum KRT. 6-8, H-1088, HUNGARY

Department of Mathematics, University of Louisville, Louisville, Kentucky 40292 